\title{
ARICA, 1950-80: LA FORMA URBANA DEL DESARROLLO Estructura y lógica de partes en el crecimiento extensivo.
}

\author{
ARICA, 1950-80: THE URBAN FORM OF DEVELOPMENT \\ Structure and the logic of the parts in the urban sprawl
}

\section{Torrent, Horacio}

(Escuela de Arquitectura, Pontificia Universidad Católica de Chile)

htorrent@uc.cl

\section{RESUMEN}

La ponencia presenta las formas que asumió la configuración del crecimiento extensivo de una ciudad de tamaño medio, empleando los instrumentos del análisis urbano para la identificación de los proyectos básicamente urbano residenciales- que propendieron a la configuración de una ciudad de partes. Arica, constituyó entre los años cincuenta y setenta, un laboratorio particular en el que se caracterizó esta relación entre arquitectura y ciudad de manera excepcional. Se presentan así los crecimientos de la planta urbana, y el análisis de una serie de proyectos de vivienda que propusieron el crecimiento expansivo, en directa relación con las lógicas internas a cada porción o parte y una lectura interpretativa del tejido, en correlación con los planes que trataron de controlar el desarrollo urbano.

Palabras clave morfología urbana, dispersión urbana, crecimiento expansivo, Arica.

Bloque temático: morfologías urbanas.

\begin{abstract}
The paper present the different configurations of the urban development growth of a medium-sized city, using the instruments of urban analysis for the identification of the projects -basically urban residential- that favored the configuration of a city of parts. Arica, between the fifties and seventies, became a privileged urban laboratory that characterized the city by the relationship between architecture and urban form in an exceptional way. The paper shows the growth of the urban plant, and the analysis of a series of housing projects that proposed expansive growth, in a direct relationship with the internal logics and an interpretive reading of the fabric, in correlation with the plans implemented to control the urban development.
\end{abstract}




\section{Introducción}

La relación entre arquitectura y ciudad, que había sido predominante en Chile desde fines de los años veinte y hasta mediados de los cincuenta del siglo XX, fue capaz de dar forma y carácter al proceso de concentración urbana y al fenómeno de crecimiento urbano poblacional, tanto en las grandes ciudades como en la intermedias. Pero desde fines de los años cincuenta esa relación fue transformada por una noción de desarrollo más abstracta y menos espacial, que promovió el crecimiento urbano por fragmentos, por paños y por partes, donde la arquitectura a la vez que incrementó su autonomía formal en los casos particulares, asumió la serie constructiva y la homogeneización por medio de la repetición de unidades ${ }^{1}$.

Esta nueva condición surgió ante la aplicación de las ideas del "desarrollo" y en particular las del "desarrollo urbano" -que asumió una dimensión múltiple de base económica y social y no solo arquitectónica-urbana fomentadas por adopción de planes más comprensivos del fenómeno urbano, por la construcción de edificios unitarios programáticamente, por la definición prioritaria de la trama vial para el crecimiento por extensión y por la construcción de grandes porciones urbanas por medio de proyectos de vivienda, superando la condición de unidad que había caracterizado el momento inicial de la relación entre la arquitectura moderna y la ciudad que se aspiraba a construir. El proceso resultó en una forma urbana caracterizada por partes desagregadas con gran autonomía.

Este formato inicial, propendió a la construcción cada vez más autónoma de grandes porciones de la periferia de las ciudades; por al menos veinte años esta forma de hacer ciudad por grandes paños o partes se asumió frecuentemente como un modelo de desarrollo urbano. Las políticas neoliberales que liberaron el limite urbano e hicieron disponible enormes extensiones de suelo urbano, convirtieron el formato de crecimiento extensivo en la operación más habitual y promovida del desarrollo urbano. El trabajo forma parte de un proyecto mayor que tiene por objetivo describir, caracterizar e interpretar la relación entre arquitectura moderna y ciudad desarrollada durante la segunda mitad del siglo veinte, con énfasis en la dimensión del desarrollo y en la configuración de las ciudades chilenas.

No obstante, la extensión de la ciudad de Arica, sucedida entre los años cincuenta y setenta, parece prefigurar una parte importante de los efectos que años mas tarde se asumirían en gran parte de las extensiones de las ciudades chilenas, incluso las áreas metropolitanas. La disponibilidad de suelo y las urgencias del crecimiento poblacional modelaron parcialmente las formas del crecimiento extensivo.

Arica, constituyó un laboratorio particular en el que se caracterizó esta relación entre arquitectura y ciudad de manera excepcional. Se trata aquí de exponer un trabajo que atiende específicamente a la identificación de las formas de configuración del crecimiento extensivo de una ciudad de tamaño medio como Arica, empleando los instrumentos del análisis urbano para la identificación de los proyectos -básicamente urbano residencialesque propendieron a la configuración de una ciudad de partes.

Se trata entonces de exponer las lógicas internas a cada porción o parte y establecer una lectura interpretativa del tejido, en correlación con los planes que trataron de controlar el desarrollo urbano, que fueron claramente superados por los proyectos particulares que dieron forma a la ciudad. Si bien el trabajo es inicialmente una apuesta de lectura gráfica e interpretativa de carácter investigativo, se trata también de afirmar las posibilidades proyectuales que el análisis tiene con vistas al proyecto urbano y su contribución a la elaboración de políticas públicas de mejoramiento barrial o urbano, que no queremos perder como referencia.

\footnotetext{
${ }^{1}$ El presente trabajo forma parte del proyecto FONDECYT No 1181290 "Arquitectura Moderna y Ciudad: obras, planes y proyectos en el laboratorio del desarrollo. Chile 1930-1980", desarrollado por el autor como Investigador Responsable. Se agradece a Fondecyt, por el apoyo otorgado.
} 


\section{La transformación de Arica entre 1950 y 1980}

Arica fue fundada en la costa del Pacífico durante el siglo XVI, con la estructura regular que caracteriza a las ciudades de la colonización española en América. Con posterioridad a la guerra del Pacífico, en 1883 pasó a ser administrada por Chile. No obstante su posición estratégica en la frontera, permaneció a su suerte por varios años con posterioridad a su integración definitiva al territorio chileno con el tratado de 1929.

Recién en 1953, se propusieron diversas estrategias de desarrollo que transformarían su base económica y establecerían sus posibilidades de progreso social. La primera de estas acciones fue el establecimiento de un régimen de franquicias tributarias para la región, conocido como puerto libre, con una zona franca y un área destinada al asentamiento industrial que se promovía. La segunda sería la creación de un organismo de carácter descentralizado y regional destinado administrar y reinvertir en la ciudad y el departamento los beneficios de las políticas tributarias. La Junta de Adelanto de Arica, establecida desde fines de 1958, desempeño un rol de liderazgo de las en pos del desarrollo local, promoviendo la planificación urbana, la realización de obras viales y de infraestructura, equipamientos públicos y conjuntos de vivienda, todos con una fuerte identidad formal basada en la arquitectura moderna.

Las expectativas generadas por estas acciones promovieron a su vez un fenómeno migratorio de importancia que duplicó la población en los ocho años inmediatos, lo que se repitió nuevamente en la década siguiente. Así la población urbana paso de 23.033 habitantes en 1952 a 46.686 en 1960, y a 87.726 habitantes en 1970.

La demanda por vivienda creció a la par que la población, sobre todo en una ciudad que contaba con mucho suelo disponible, pero muy pocas posibilidades de llevar adelante grandes inversiones en vivienda, a la vez que no contaba con un mercado inmobiliario, y las posibilidades de autoconstrucción estaban muy limitadas por su posición en el desierto. Este proceso acelerado puso en alerta a la ciudad y a las administraciones públicas que llamaron reiteradamente la atención sobre el problema. Durante la década del 50 , la construcción de viviendas fue llevada a cabo por la Corporación de la Vivienda - de nivel nacional- y la Sociedad Modernizadora de Arica, creada por la Corporación Nacional de Inversiones como un ente descentralizado que pudo trazar algunas definiciones propias respecto a la forma de operar en relación con la vivienda. Desde 1958, la labor estuvo a cargo de la Junta de Adelanto en coordinación con corporaciones y entes estatales, como las EMPART, la CORVI, la CORMU, entre otras.

El enorme crecimiento poblacional indujo la consecuente demanda de vivienda sin distinción de sectores sociales. La disponibilidad de suelo fiscal provocó la expansión de la planta urbana en un tejido inicialmente bastante uniforme donde los diferentes de conjuntos de vivienda o poblaciones se generaron como proyectos unitarios determinando diferencias en el trazado de enormes paños de suelo, que solo asumieron la vialidad primaria prevista en los planes urbanos.

\section{El control de la forma urbana: planes y procesos}

No fueron pocas las propuestas de regulación de la forma urbana durante el período. Un inicial plan urbano habría sido realizado hacia los años cuarenta en la Sección Urbanismo de la Dirección General de Obras Públicas -y del cual se tiene muy poca noticia- establecería una regulación urbana preliminar. Sin lugar a dudas sus previsiones fueron desbordadas cuando las expectativas de florecimiento de la economía generadas por la figura del Puerto Libre dieron lugar a un fenómeno migratorio que provocó el crecimiento inaudito de la población durante las dos década subsiguientes. Este fenómeno y la consecuente demanda de vivienda provocaron a su vez una acelerada expansión de la planta urbana con destino a vivienda. 
Las propuestas de regulación asumieron claramente tres desafíos: la presión sobre el centro provocada por el aumento de los equipamientos y servicios, la disposición de las superficies destinadas a la industria y la expansión de los tejidos residenciales.

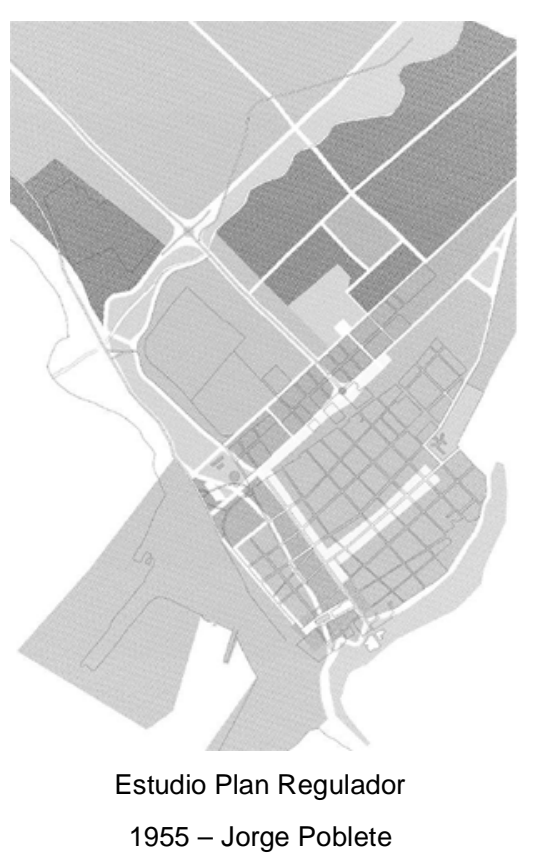

Fig. 01 Propuestas de planes reguladores de Arica entre 1955 y 1958. Elaboración FONDECYT 1181290 sobre información provista por Centro de Documentación MINVU.

La puesta en marcha del puerto libre y la zona franca, determinaron claramente la necesidad de una zonificación que demarcara las porciones de suelo que podían ser ocupados por usos industriales. En 1954, al año siguiente del establecimiento del puerto libre, Jorge Poblete Grez realizó el plan regulador que determinó la zonificación y la vialidad, los dos problemas claves para enfrentar el futuro urbano en relación a su nueva condición regional y nacional. Este plan estableció la localización industrial hacia el norte de la planta urbana, y una vialidad que la conectara con el puerto. La expansión prevista para la zona residencial quedó limitada al área entre la ciudad existente y la zona industrial, lo que a poco andar se reveló como insuficiente. Ya en 1957, Osvaldo Hufe revisaría la propuesta en el marco de la Dirección de Planeamiento del MOP, ampliando el área de expansión residencial hacia el oriente, pero manteniendo la limitación impuesta por la localización de la zona industrial. En 1958, estos estudios fueron retomados por Amador Brieva y Ventura González, quienes acotaron la zona industrial, mantuvieron sin mayores cambios la jerarquía de la vialidad y establecieron zonas residenciales según destinaciones socio económicas. Este plan se mantuvo vigente por bastante tiempo, junto con la Ordenanza Local que lo especificaba. Posteriormente, algunos intentos de revisión estuvieron constituidos sucesivamente por el diagnóstico de Federico Oherens, la propuesta de Guillermo Jones Odriozola, realizada como consultor de Naciones Unidas de 1964, y la de Emilio Duhart de 1969, sin que ninguna fuera definitivamente aprobada.

Si bien, en esta ponencia, el análisis no profundiza plenamente en las diferentes propuestas anotadas, resulta evidente que la ciudad fue creciendo más allá de las previsiones de los instrumentos planificadores. Los crecimientos de la planta urbana que hemos logrado determinar -aún parcialmente- en base a documentación de operaciones parciales constituidas por loteos, poblaciones y conjuntos habitacionales, muestra claramente que la necesidad de vivienda y los proyectos particulares para responder a esa demanda superaron con creces las previsiones que la planificación propuso en los planes reguladores o sus estudios. 


\section{SIIUU}

Los proyectos particulares de vivienda superaron las previsiones de los planes reguladores vigentes y se realizaron en su mayoría por medio de extensiones parciales del límite urbano y con la figura del plan seccional. Pero cómo lo hicieron en su morfología, puede entenderse a través de la presentación de varios de los casos, tanto en la generación de tejidos urbanos modernos, como por la extensión de tejidos residenciales principalmente formados por la repetición de unidades.

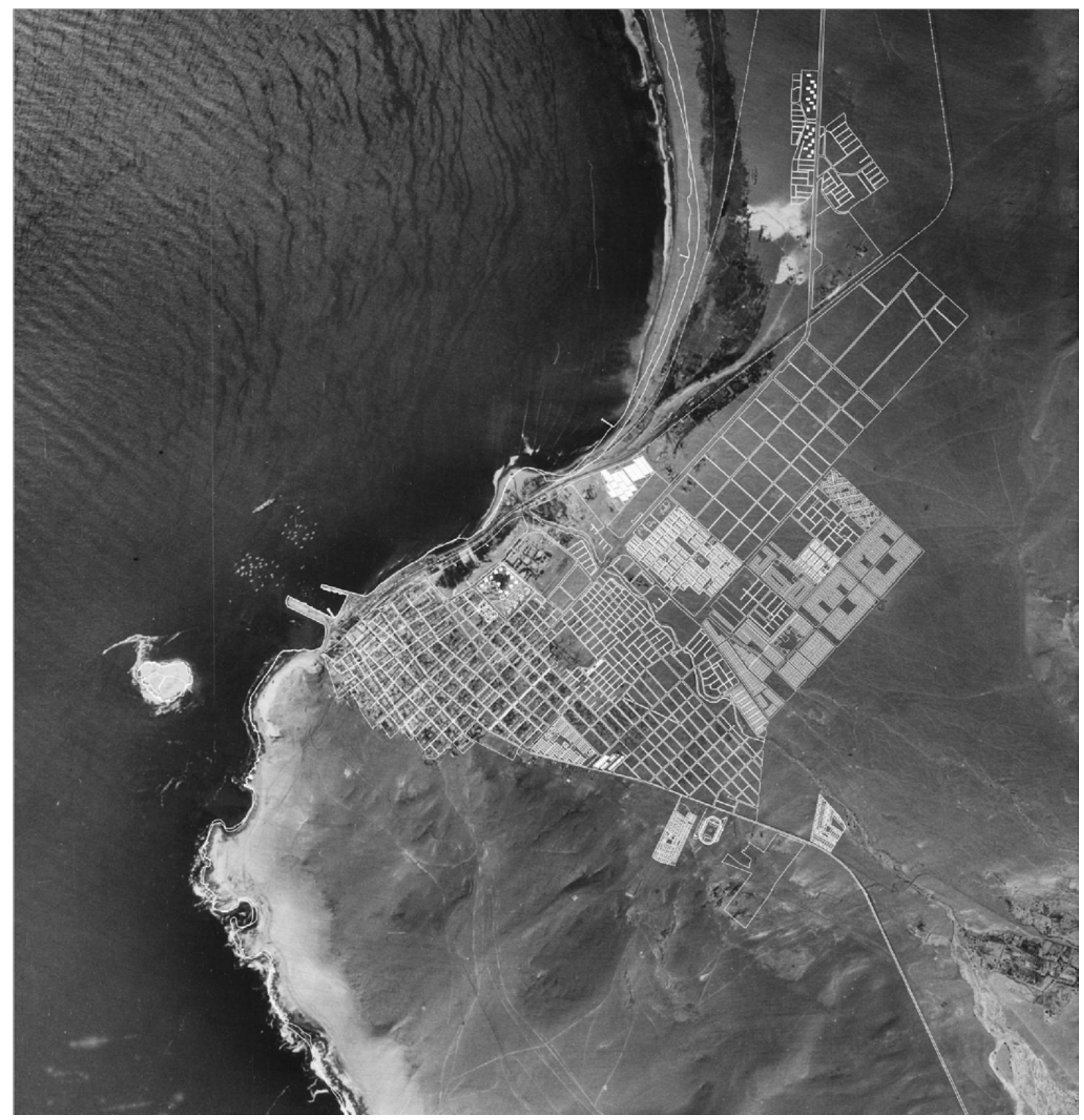

Fig. 02 Crecimientos urbanos por sectores poblacionales entre 1953-1971 (avance parcial). Elaboración FONDECYT 1181290 a partir de planimetría de la DOM de la Municipalidad de Arica y fotografía aérea del IGM de 1953.

\section{Proyectos habitacionales: configuración de tejido urbano moderno.}


La necesidad de vivienda puso en debate los dos modelos más habituales del urbanismo de ese tiempo: la densificación por medio de tipologías básicas -como el bloque extendido o el cuerpo cuadrado- y expansión por medio de unidades de vivienda aisladas, pareadas, o en línea, cubriendo como un extenso tapiz el suelo del desierto.
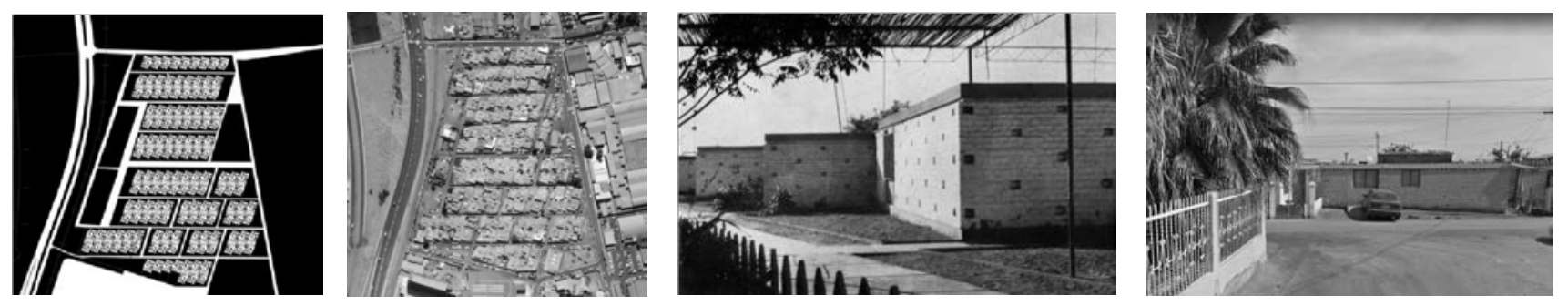

Fig. 03 Población Chinchorro. Planta conjunto, fotografía aérea actual, fotografía de época y fotografía actual. Plano elaboración FONDECYT 1181290 a partir de planimetría de la DOM de la Municipalidad de Arica y fotografía aérea del IGM de 1953. Fotografías actuales Google Earth, fotografía 1956 R. Combeau, archivo de originales SLGM, PUC.

La Población Chinchorro fue proyectada por Bresciani, Valdés, Castillo y Huidobro, junto a Arnello, Lorca y Jordán, en 1955. Se configuró en base a la repetición de unidades elementales -casas con patio- que se desplazan sesgadas respecto al trazado regular de la estructura vial primaria. Se disponían en una secuencia de volúmenes puestos en situación oblicua respecto a los pasajes, proponiendo una serie de pequeños espacios públicos.

La población Ex Estadio combinaba bloques y casas y fue proyectada también por Bresciani, Valdés, Castillo y Huidobro entre 1956 y 1957 . La pendiente del terreno orientó algunas de las decisiones de proyecto y la cercanía al centro de la ciudad, definió la posibilidad de dar mayor altura y mayor densidad al conjunto. Así, una serie de bloques dispuestos sesgadamente en el sitio se orientaban hacia el mar y el mejor asoleamiento, definiendo un área central. Los bloques tenían entre cinco y seis pisos asumían en su definición las relaciones con la topografía, y sus calles elevadas se relacionaban directamente con los niveles de la calle. El conjunto alterna los bloques con casas dispuestas en el borde superior del sitio, que por la pendiente, se articulaban espacialmente en diferentes niveles entre si y con los bloques aledaños. La trama esta orientada a 45 grados respecto de los límites del terreno, aseguraba una mayor variedad de situaciones espaciales.

El caso probablemente más paradigmático de la combinatoria sea el de la Población Lastarria, un superbloque de XX metros de largo, acompañado por 21 viviendas con patio, que la Junta de Adelanto realizó destinado a su propio personal. El superbloque, de unos 130 metros, recorre la casi totalidad de la dimensión del sitio, en seis pisos, organizándose con 78 unidades con dúplex y departamentos de varias dimensiones. Las 21 casas fueron dispuestas giradas respecto a la calle, traslapadas de modo de ir formando una secuencia de planos diagonalizados con la pretensión de entregar perspectivas mas interesantes acompañando el largo del superbloque.

Pero el laboratorio de alternativas conjuntas de densificación y extensión quedó limitado y las definiciones posteriores fueron tomadas en el amplio abanico de posibilidades que fueron permitiendo tanto las diferentes agencias involucradas, como la CORVI y la CORMU, y siguiendo incluso algunas búsquedas particulares que determinaron sus características.

De unos pocos casos analizados hasta el momento es posible determinar que la estrategia de desarrollo urbano adquirió formatos opuestos según localización. Para los proyectos realizados en suelos que eran relativamente centrales, se optó por realizaciones que asumían condiciones de mayor densidad, en bloques o cuerpos compactos y a la vez liberaban mayor cantidad de suelo, siguiendo las concepciones de la arquitectura 
moderna. Así por ejemplo, el conjunto habitacional Pucará, proyectado por Juan Galleguillos, Hernán Rodríguez y Florentino Toro para la CORMU en 1968, se configuró por doce bloques o cuerpos cúbicos, de planta casi cuadrada, emplazados en un sector en pendiente. Estos cuerpos tenían 5 pisos y cuatro departamentos por piso, con un sistema de escaleras a medios niveles y a los que por lo general se ingresaba por el primer piso o piso y medio, por medio de una escalera o rampa que los conectaba al mismo nivel con las vías principales.
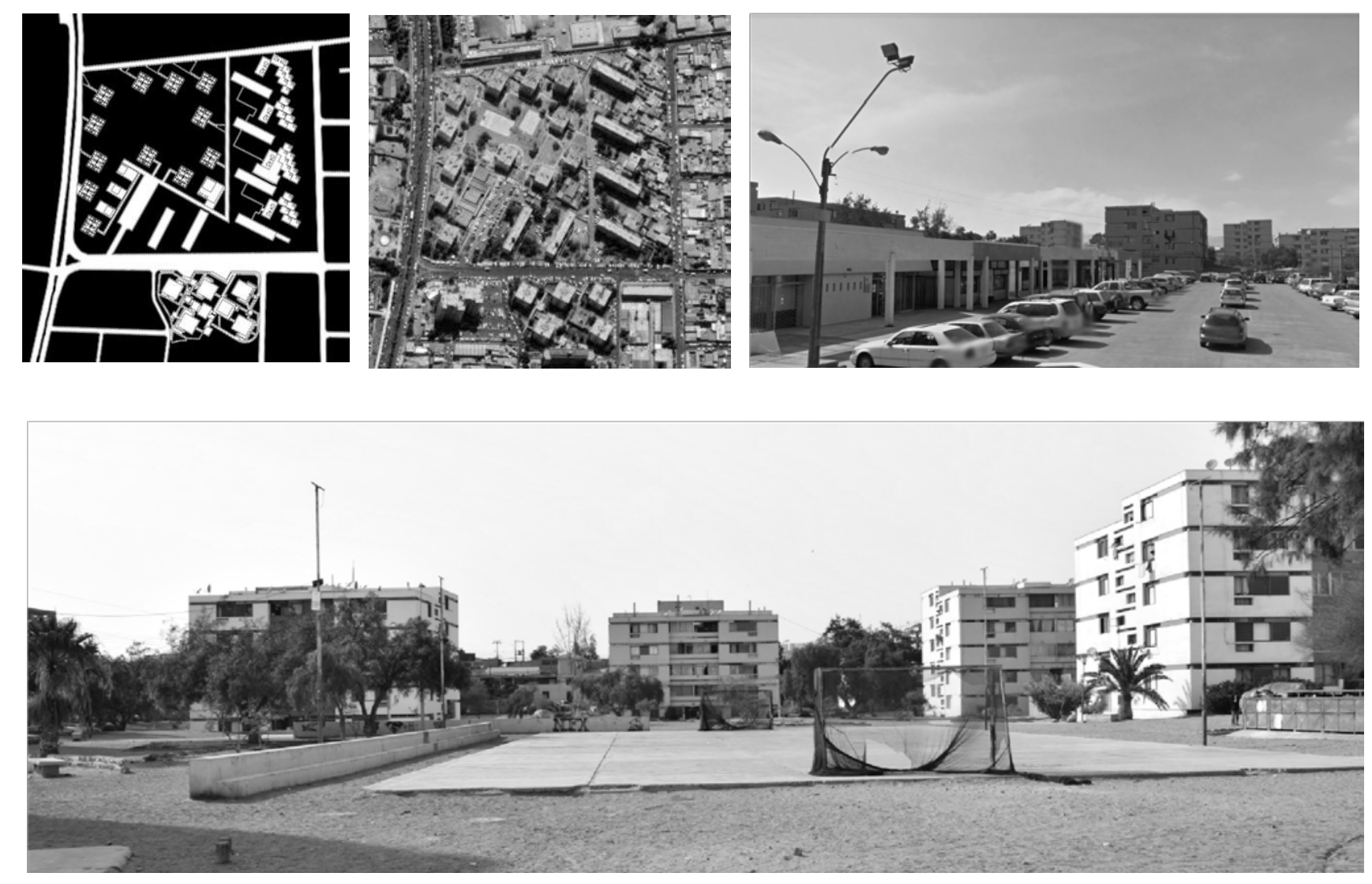

Fig. 04 Poblaciones Pucará, Chungará y Ex Estadio. Planta conjunto, fotografía aérea actual y fotografías del proyecto. Plano elaboración FONDECYT 1181290 a partir de planimetría de la DOM de la Municipalidad de Arica y fotografía aérea del IGM de 1953. Fotografía 1 Google Earth, Fotografía 2 Javier Ruiz FONDECYT 1181290

En un sistema bastante similar, el Conjunto Habitacional Chungará fue proyectado por Anselmo Trepiana y Sergio Román en 1971, también para la CORMU. Está compuesto por cinco cuerpos de planta cuadrada, dos de ellos de 5 pisos y tres de 7 pisos de altura respectivamente. Tambien situados en pendiente, un sistema de recorridos, pasarelas, rampas y escaleras conecta los bloques entre sí y con un espacio público central. En los bloques de 5 pisos, los accesos se encuentran en los medios niveles 1 y 4 , mientras que en los de 7 pisos lo hacen en los medios niveles 3 y 6 , por lo que el sistema de conexiones no se desarrolla en un mismo nivel sino que en diferentes alturas, conectando también las vías perimetrales.

\section{La extensión de los tejidos residenciales: poblaciones de casas.}

La gran diferencia se produjo en los suelos que se disponían mas allá de la zona central de la ciudad y sobre todo en relación con la larga faja de la zona industrial. Paulatinamente, pasado el límite del río, las poblaciones adquirieron básicamente la configuración de una urbanización de pequeñas manzanas y pasajes, que variaban según el proyecto y la forma de gestión, incluyendo a veces la edificación y otras asumiendo variaciones. 

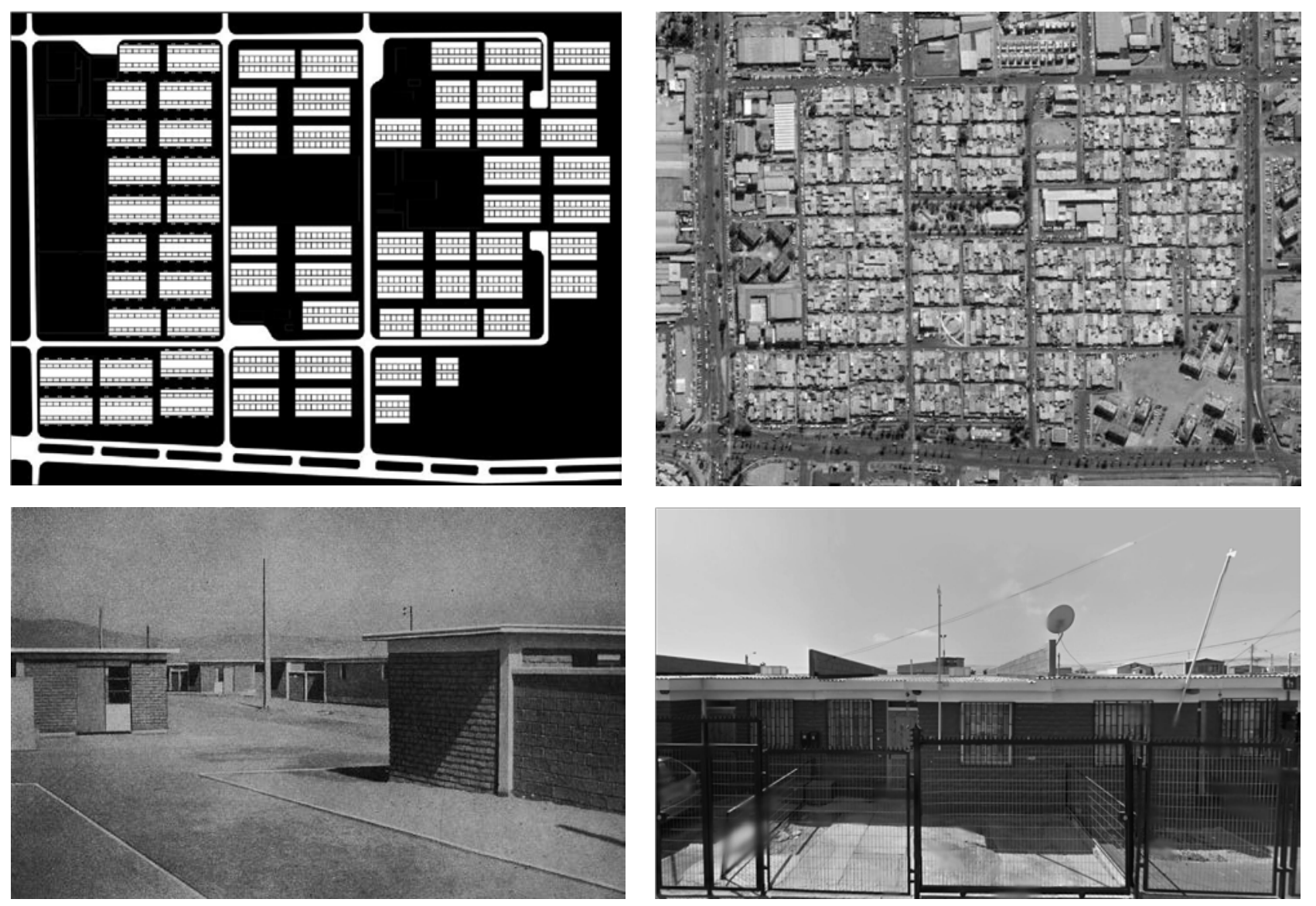

Fig. 05 Población Juan Noé. Planta conjunto, fotografía aérea actual, fotografía de época y fotografía actual. Plano elaboración FONDECYT 1181290 a partir de planimetría de la DOM de la Municipalidad de Arica y fotografía aérea del IGM de 1953. Fotografías actuales Google Earth. Fotografía de época en Rebolledo G.; Rivera, H. Población Juan Noé. Plan Habitacional Chile Coorporación de la Vivienda, 1959.

La Población Noé proyectada en el taller de la CORVI por Gloria Rebolledo y Hernán Rivera en 1959-60 inauguró tempranamente el crecimiento expansivo sobre la base de un trazado de manzanas alargadas y pasajes con un modelo de vivienda económica. Constituida por con 1056 viviendas en un gran paño de 25 hectáreas que se subdividió por medio de un trazado lineal perpendicular a las grandes avenidas. Las manzanas se generaron por la repetición de unidades, dejando en el centro un área para equipamientos, así como también sobre uno de los bordes.

Esta población preanuncia un cambio de importancia en la definición de la forma urbana, dejando de lado los aspectos más radicalmente modernos que se habían ensayado y proponiendo una extensión homogénea de viviendas de un piso, una condición que se asumiría -salvo excepciones- en los proyectos de muchas poblaciones posteriores. 


\section{SIIU}

barcelona|santiago de chile junio 2019
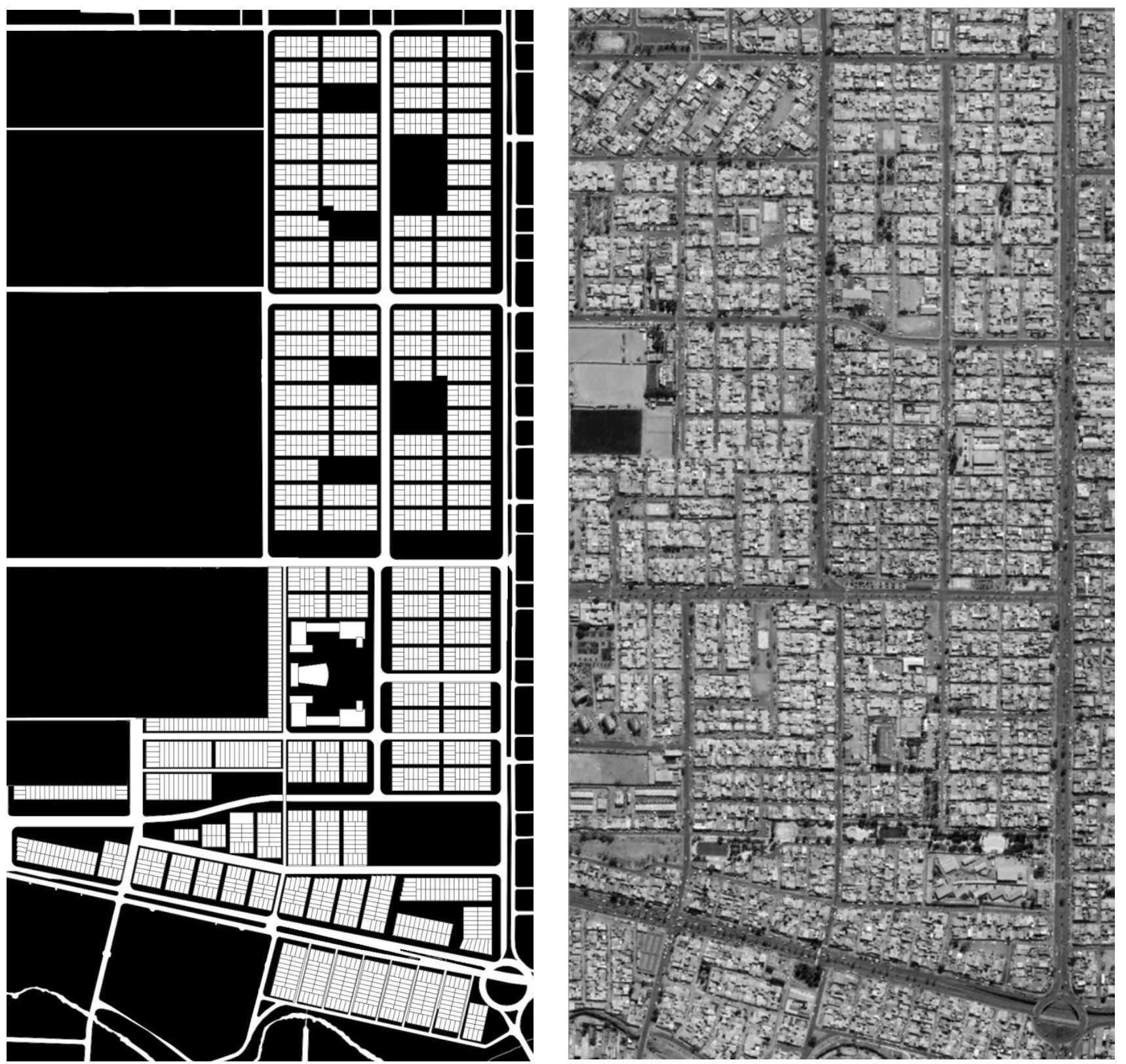

Fig. 06 Población San José. Planta conjunto y fotografía aérea actual. Plano elaboración FONDECYT 1181290 a partir de

planimetría de la DOM de la Municipalidad de Arica y fotografía aérea del IGM de 1953. Fotografía Google Earth.

La Población San José fue proyectada en el Departamento Técnico de la JAA, por Gastón Saint Jean, Patricio Moraga, Jorge Vallejo la primera etapa, sumándose posteriormente Federica Frank, en 1961, bajo la forma de una urbanización por loteo cuya primera etapa constó de 56 y la segunda de 72 manzanas. Los lotes de 9 × 18 metros, generaron un tipo de manzana con cuatro lotes en el cabezal, variando rectángulos entre los $81 \times 36$ y los 72 × 36 metros, con pasajes de entre 6 y 8 metros. Una base por demás de homogénea y que establecía restricciones muy claras a la ocupación de los cabezales de manzana. El loteo destinó espacios para áreas verdes y equipamientos comunes como iglesia, escuela, mercado y un centro cívico y comercial; con una manzana libre para un matadero frigorífico, un garaje y la ganadera Portales. En la segunda etapa, tres manzanas estaban destinadas a áreas verdes y dos de mayores dimensiones, una para un centro comercial y otra para una escuela. 


\section{SIIU}

barcelona|santiago de chile junio 2019
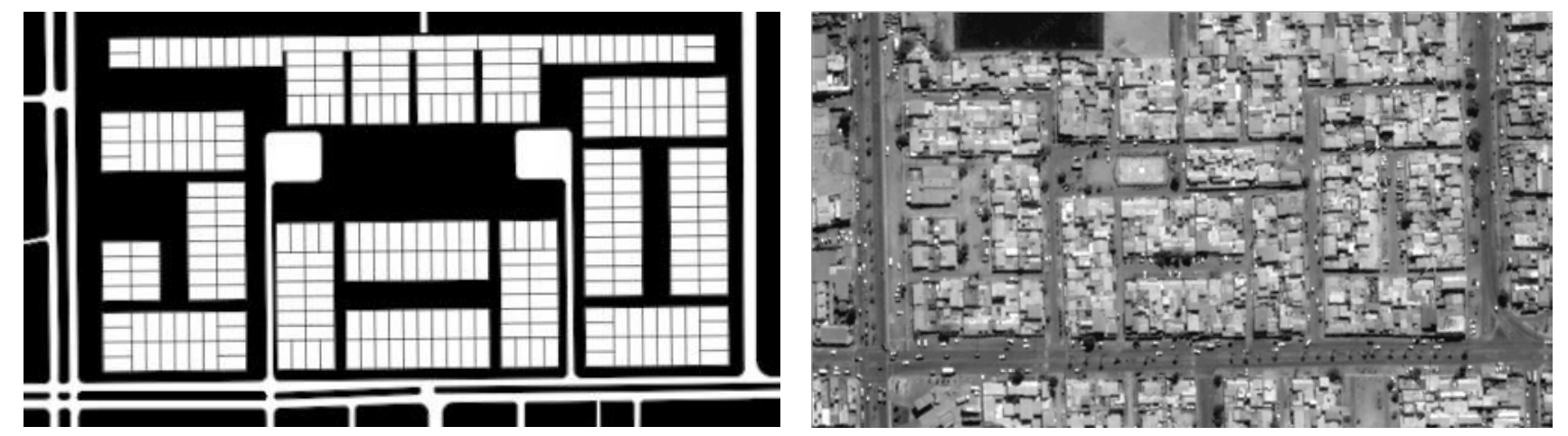

Fig. 07 Población Radio El Morro. Planta conjunto y fotografía aérea actual. Plano elaboración FONDECYT 1181290 a partir de planimetría de la DOM de la Municipalidad de Arica y fotografía aérea del IGM de 1953. Fotografías aéreas y actuales Google Earth.

La población Radio El Morro fue proyectada por Sergio Moreno, Walterio González en el marco de la CORVI en 1971 y consistió en un loteo organizado inicialmente en una supermanzana con dos entradas vehiculares, aunque con pasajes interiores que daban lugar a trece manzanas, doce de ellas de dimensiones similares según la disposición de lotes 9 × 18 (con 20 a 30 casas), mientras que el último conforma el fondo del sitio, por lo que toma la extensión de la manzana.
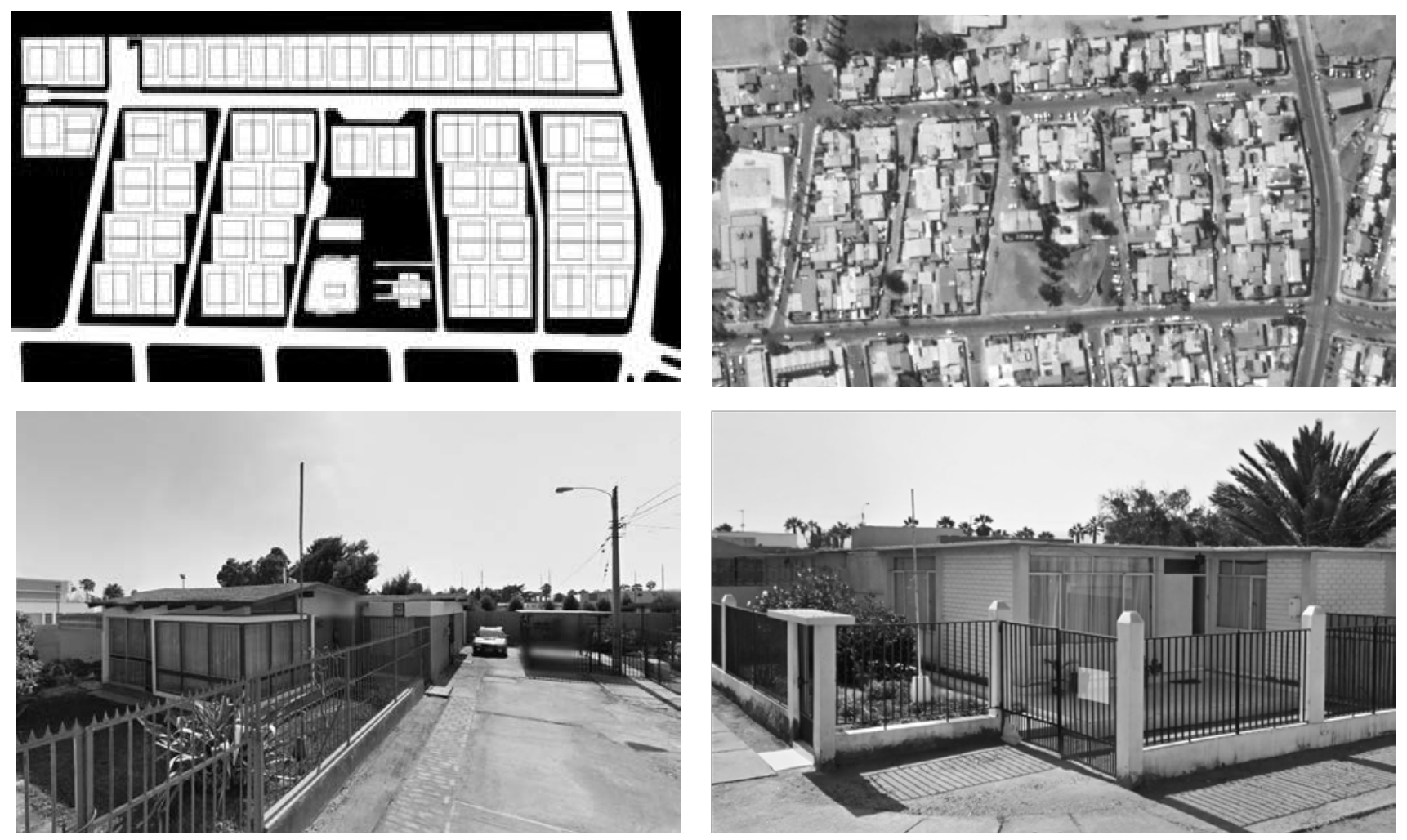

Fig. 08 Población Simón Bolívar. Hermógenes Pérez, Nelson Berthelon (CORMU), 1968. Planta conjunto y fotografía aérea actual. Elaboración FONDECYT 1181290 a partir de planimetría de la DOM de la Municipalidad de Arica y fotografía aérea del IGM de 1953. Fotografía aérea y fotografías actuales Google Earth.

La Población Simón Bolívar proyectada por Hermógenes Pérez y Nelson Berthelon en la CORMU, 1968, consiste en un loteo de 6 manzanas (5 manzanas completas, una cabeza de manzana y 8 sitios adyacentes) situados al lado del estadio Carlos Dittborn. La manzana central deja espacio libre para juegos infantiles, un parvulario y comercio. De manera posterior aparece un proyecto de conjunto residencial para 7 viviendas de la 
población, propiedad de la Caja de Previsión del Banco del Estado, del arquitecto Alberto Sainte-Marie Pressac. Las casas tipo incorporadas a la población fueron proyectadas por Eduardo Hoyos, Hernán Lagos y Patricio Moraga en 1968.

La Población Alejandro Azolas puede representar claramente un proceso que parece haber sido frecuente en la gestión del desarrollo urbano. En un primer momento, la urbanización fue proyectada por Hermógenes Pérez en la CORMU en 1968, generando un trazado de unidades combinadas, consistente en 8 manzanas con sitios de $10 \times 20 \mathrm{~m}$,. Sobre ese trazado de base, otros agentes públicos y privados asumían la construcción de proyectos de vivienda, en algunos casos bajo formas de distribución que implicaban unidades urbanas y en otros simplemente disponían de lotes aislados de diferentes localizaciones para la construcción de idénticas tipologías.
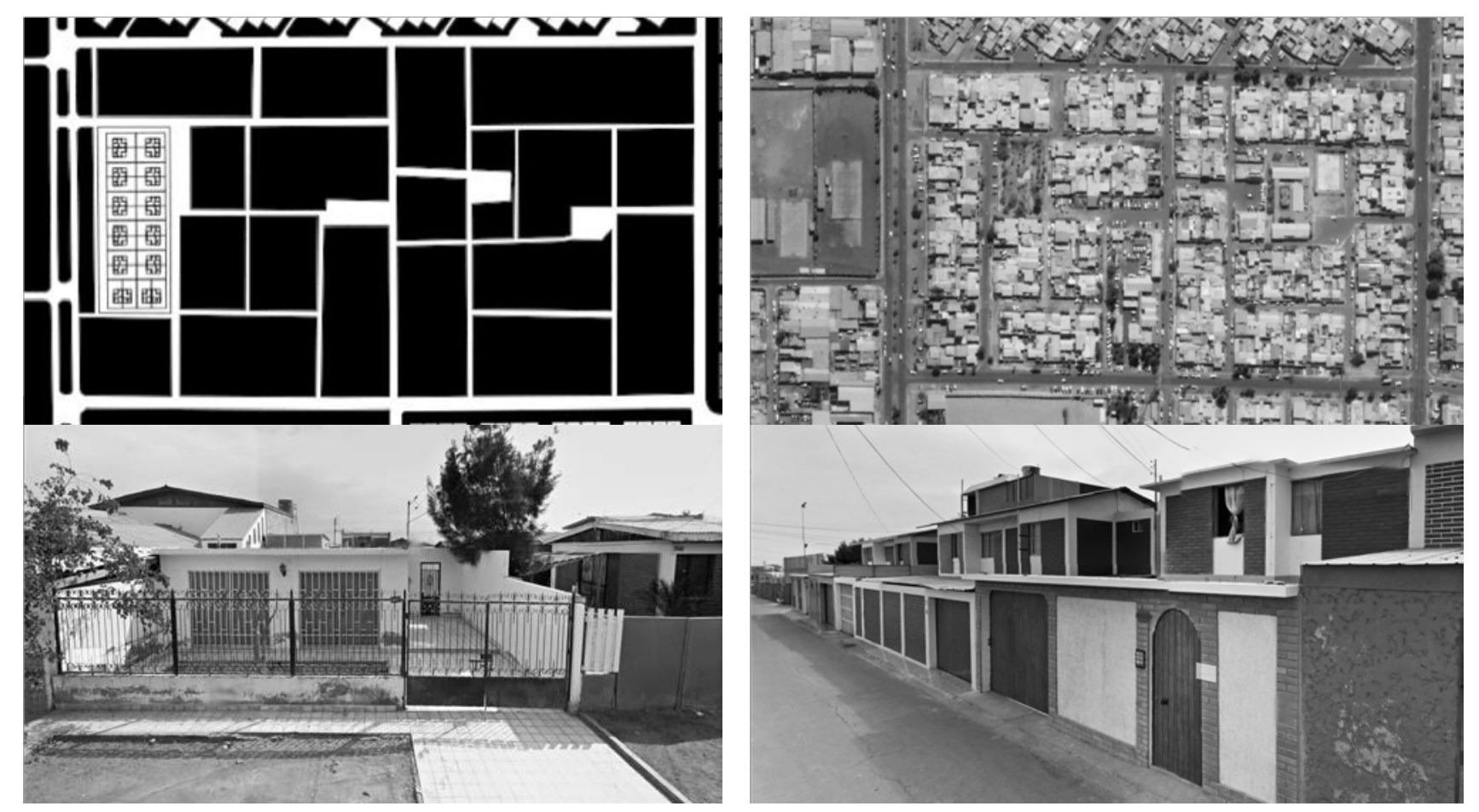

Fig. 10 Población Alejandro Azolas. Planta conjunto y fotografía aérea actual. Elaboración FONDECYT 1181290 a partir de planimetría de la DOM de la Municipalidad de Arica y fotografía aérea del IGM de 1953. Fotografía aérea y fotografías actuales Google Earth.

La unidad de trazado urbano base, fue una pequeña manzana más larga que ancha, con lotes en uno de los cabezales y cierre lateral en el otro. Se presentaba como una unidad con relativa indiferencia respecto de la totalidad del tejido y del espacio urbano resultantes. La modalidad de gestión parcial, producto de una demanda particular, fue en un caso respondida a través de un proyecto realizado en 1971, consistente casas pareadas de $45 \mathrm{~m} 2$ denominadas Aricasas, se construyeron en 5 manzanas, para dar tan solo un ejemplo. 


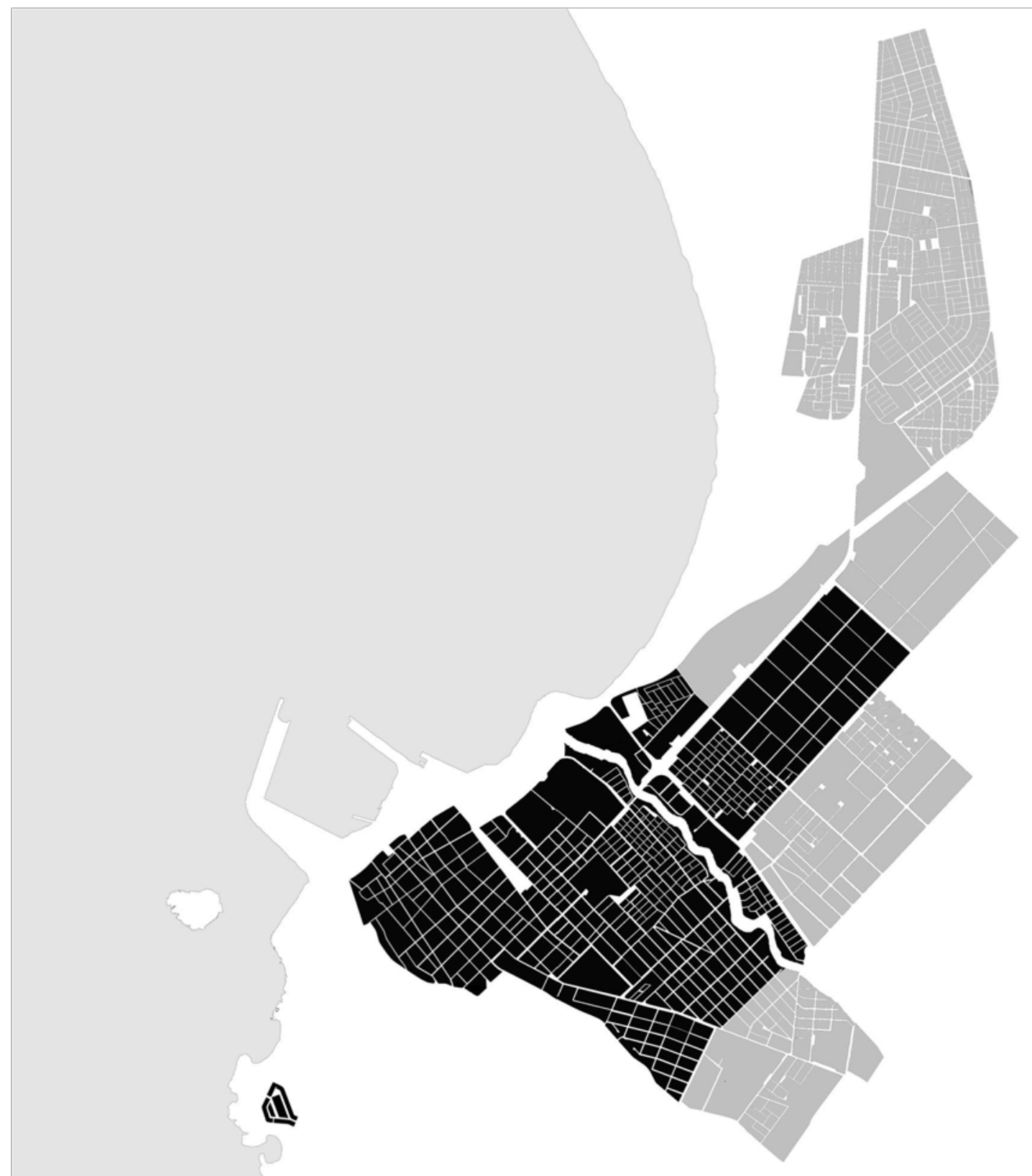

Fig. 11 Zonas de crecimiento fuera (gris) de las propuestas de planes reguladores de Poblete (1955), Hufe (1957) y Ventura González (1958), entre 1959 y 1970 aproximadamente. (plano en desarrollo sobre verificaciones de permisos de edificación). Elaboración FONDECYT 1181290 a partir de fotografía aérea del IGM de 1953. planimetría y permisos de edificación del Archivo de la DOM de la Municipalidad de Arica.

\section{Crecimiento extensivo y partes de ciudad: un análisis en curso}

La construcción paulatina de un plano que registre la secuencia de incorporación de las diferentes poblaciones, es en sí mismo un plano de los crecimientos urbanos.

Hace tiempo ya que se pusieron de manifiesto las ventajas del estudio de los crecimientos, entre otras porque ofrecen "una aprehensión global de la aglomeración urbana en una perspectiva dinámica, seguida de precarias estabilidades basadas en la cohesión interna de la ciudad a las que suceden períodos de ruptura y saltos en el desarrollo, no suponiendo el estado actual más que un momento de esta evolución" (Panerai 1984:28). El trabajo sistemático de la incorporación de las poblaciones en el dibujo del plano va revelando las diferencias 


\section{SIIU}

entra cada parte concebida como un proyecto diferente. Ya hemos advertido que la continuidad aparece dada por el trazado de las vías primarias previstas en la figura del plan regulador.

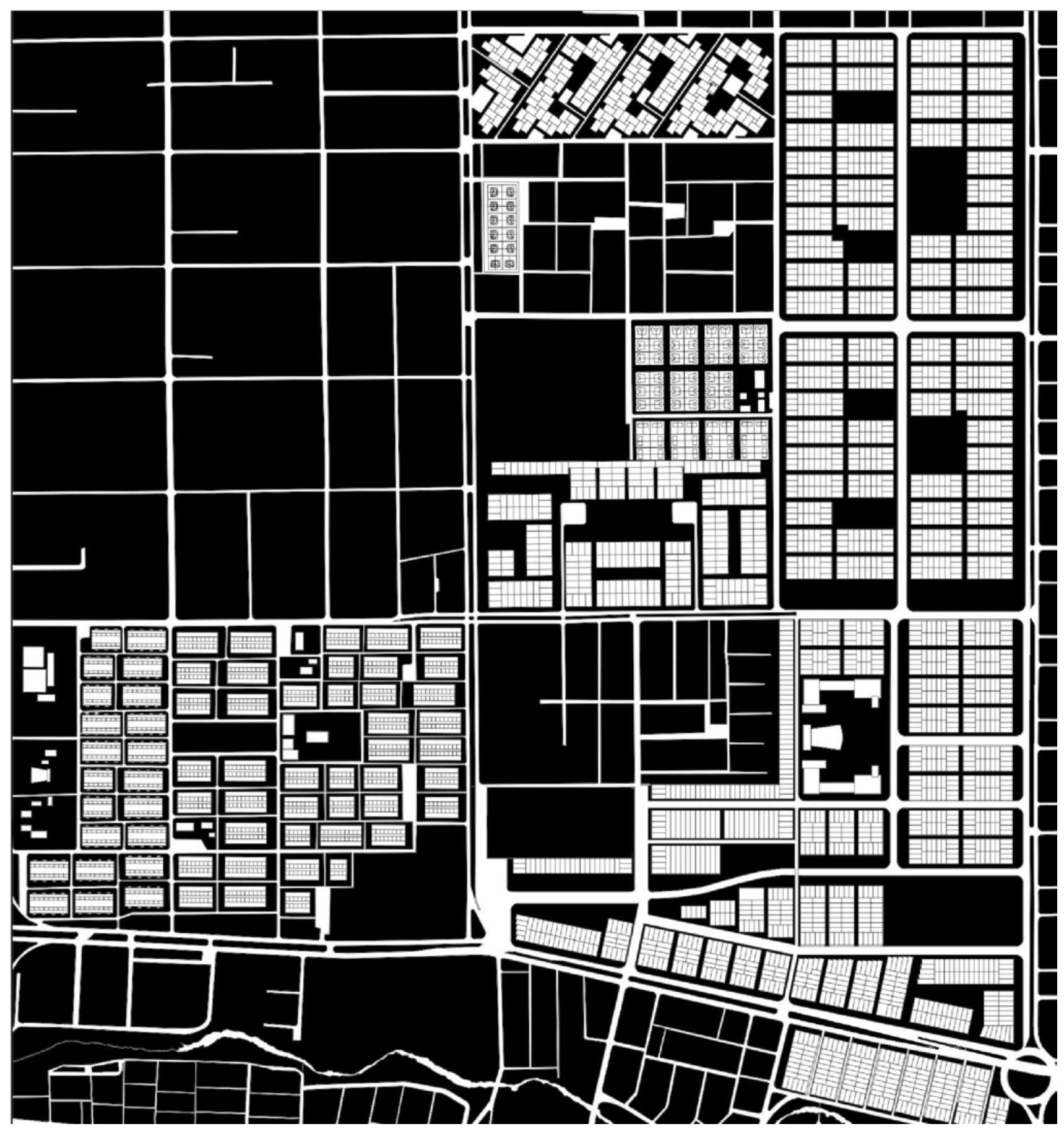

Fig. 12 Configuración de partes urbanas. Elaboración FONDECYT 1181290 a partir de planimetría de la DOM de la Municipalidad de Arica y fotografía aérea del IGM de 1953.

La parte a la vez revela algunas posibles nuevas hipótesis sobre las formas en que se fue adecuando paulatinamente el plan regulador en función del desarrollo de planes reguladores seccionales que en realidad surgían de un proyecto específico realizado para paliar la necesidad de vivienda. 
El plano revela claramente como la organización del centro urbano previa a la aplicación de las políticas de desarrollo es de crecimiento continuo, es decir la extensión de la planta urbana se da por la prolongación de las mismas condiciones ya vigentes en las partes ya constituidas de la ciudad.

En la extensión urbana posterior a la aplicación de las estrategias de desarrollo, es claramente visible la alternancia de definiciones muchas veces aproximadas pero que dada sobre las guías de la vialidad básica general, se dio un proceso acelerado de construcción urbana por medio de partes diseñadas unitariamente, con baja relación entre las propiedades de los trazados menores o internos, e incluso en los parcelamientos. Se nota así la discontinuidad del tejido urbano en tanto cada nueva parte inaugura- en mayor o menor medidauna nueva forma de relación con lo preexistente. La yuxtaposición de los diferentes trazados es evidente, y la unitariedad de cada parte también, cualquiera sea el tamaño de la intervención.

El análisis de los proyectos de las poblaciones o conjuntos habitacionales recurre a las posibilidades que brinda el análisis tipo- morfológico y estructural de modo que se dibujan, como se ha visto parcialmente, las condiciones del trazado, los límites, condiciones topográficas y las tramas de composición urbana; los sistemas viales, calles y espacios públicos predominantes; la organización de manzanas, cuadras, islas de edificación; el parcelamiento y la edificación. Sobre una selección inicial, hemos ido caracterizando los diferentes paños o partes del tejido, y se poniéndolos en relación con las dimensiones del crecimiento expansivo de la planta urbana, con el fin de construir a partir de este nuevo material, una serie de dibujos analíticos que identifican las estrategias y los instrumentos proyectuales utilizados por el urbanismo y la arquitectura.

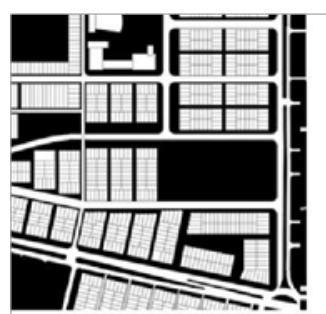

San José

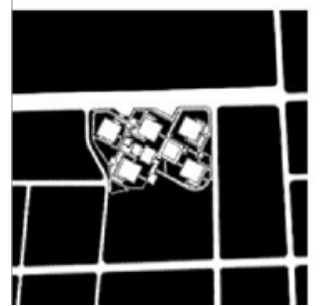

Chungará

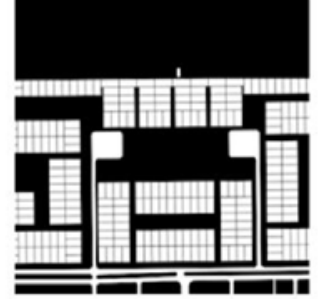

Radio El Morro

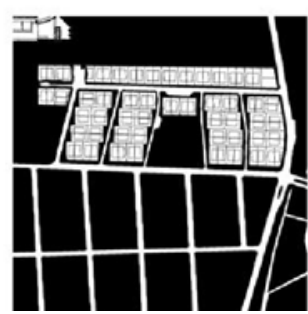

Simón Bolívar

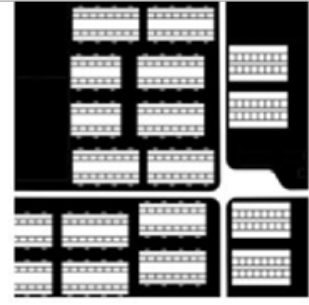

Juan Noé

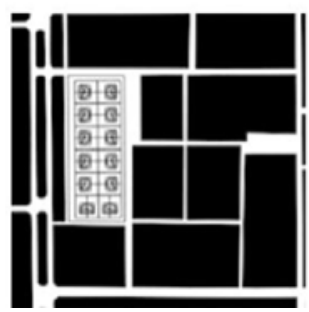

Alejandro Azolas

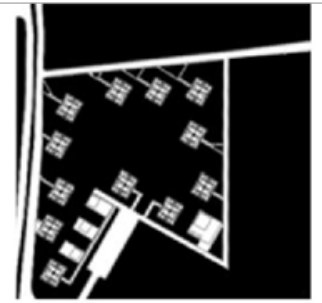

Pucará

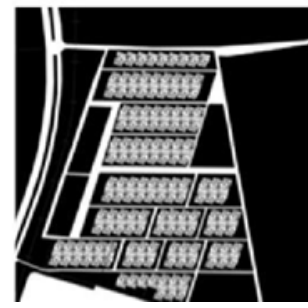

Chinchorro

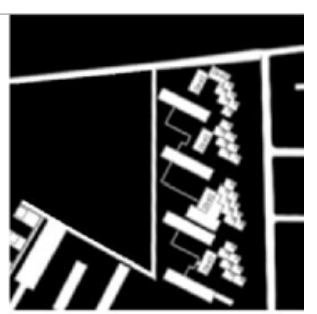

Ex Estadio

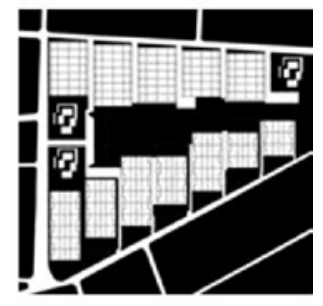

O'Higgins

Fig. 13 Trazados y parcelaciones. Esquema comparativo a escala. Elaboración FONDECYT 1181290 a partir de planimetría de la DOM de la Municipalidad de Arica y fotografía aérea del IGM de 1953.

La secuencia gráfica da cuenta de las variaciones que se han expuesto caso a caso y constituye una muestra que no pretende representatividad genérica del tejido sino que contrasta los diferentes trazados y posibles ocupaciones marcando claramente las diferencias de los proyectos en cuanto a los espacios públicos y sus continuidades. Así se da cuenta también de las lógicas internas de cada porción o parte de ciudad en que se constituyó cada población o conjunto residencial.

Si bien por el momento nuestro trabajo se propone sobre las estructuras del tejido solo parcialmente, asumiendo por ahora el trazado y las formas de la subdivisión, es posible aproximar algunas conclusiones preliminares: que las apuestas por el desarrollo tuvieron correspondencia con la conformación de un tejido urbano que solo mantenía continuidad en las grandes vías de circulación que por otra parte estaban dispuestas en el plan 
regulador; que las apuestas por el desarrollo asumidas en condición perentoria, fueron definiendo las figuras de la planificación por medio de proyectos específicos. Asimismo, que se hacen evidentes las partes tanto a nivel formal como a nivel del espacio urbano, afectando la continuidad espacial, la legibilidad y la identidad urbanas.

Y finalmente que, en tanto la expresión física del tejido es indisociable de los procesos que les dieron origen, por tanto la forma urbana estuvo determinada por proyectos parciales dando origen a un crecimiento extensivo indiferenciado por partes discretas muchas veces disociadas de la pretensión de una ciudad configurada sobre una aspiración común.

\section{BIBLIOGRAFÍA}

BRAUN MENÉNDEZ, R. (1962) Bresciani, Valdés, Castillo, Huidobro. Buenos Aires: Instituto de Arte Americano e Investigaciones Estéticas.

CHILE, CORPORACIÓN DE LA VIVIENDA (1963) Plan Habitacional de Chile. Santiago: CORVI.

MANGIN, D., PANERAI, PH. (2006) Projet urbain. Marseille: Éditions Parenthèses.

LANG, A. Puerto libre de Arica. Santiago de Chile: Tesis de Licenciatura, Universidad de Chile, 1962.

PANERAI PH. (1984) Crecimientos. En: PANERAI, PH., DEPAULE, J-C., DEMORGÓN M., y VEYRENCHE, M. (1983) Elementos de Análisis Urbano. (25-56) Madrid : Instituto de Estudios de Administración Local .

PÉREZ, F. (2006) Bresciani Valdés Castillo Huidobro. Santiago, Chile : ARQ.

PÉREZ, H. (1967) El Plano Regulador y el Desarrollo Urbano de Arica. En COLEGIO DE ARQUITECTOS. IV Convención Nacional del Colegio de Arquitectos de Chile. Santiago: Colegio de Arquitectos.

PORTAS, N (2012) Os Tempos das Formas - Livro 2: A Cidade Imperfeita e a Fazer. Minho: Universidade do Minho.

RUZ, R; GALDAMES, L; DíAZ ARAYA, A. (2015) Junta de Adelanto de Arica (1958-1976). Experiencia, Documentos e Historia Regional. Arica: Ediciones Universidad de Tarapacá.

SOLÁ-MORALES, M. (1974) Las formas de crecimiento urbano : urbanística I : curso 1973-74. Barcelona: Edicions UPC.

SOLÁ-MORALES, M. (1989) The Culture of Description. En Perspecta Vol. 25, pp. 16-25

SOLÁ-MORALES, M. (1997) Las formas del crecimiento urbano. Barcelona: Edicions UPC.

TORRENT, H. (2015) El patrimonio moderno en la construcción del país urbano: Dialécticas entre arquitectura y ciudad en Chile 1930-1970, AUS (Valdivia), 17,11-17.

TORRENT, H. (2018) Modern Heritage and the Challenge of Urban Conservation: Between Singular Buindings and the Metamorphosis of the Urban Fabric. En Tostões, A. y Koselj, N. Metamorphosis. The Continuity of Change, (140-146) Lisboa - Ljubljana: Docomomo International - Docomomo Slovenia.

TORRENT, H.; RUZ, R.; MORÁN B. (2018) Arquitecturas para la institucionalización del desarrollo: tres dimensiones en la obra de la Junta de Adelanto de Arica. En TORRENT, BARRÍA et al. (eds.), Patrimonio Moderno y sustentabilidad: de la ciudad al territorio, (124-128.) Valdivia: Docomomo Chile - Universidad Austral. 